\title{
Cost Effectiveness of Pembrolizumab vs. Standard-of-Care Chemotherapy as First-Line Treatment for Metastatic NSCLC that Expresses High Levels of PD-L1 in the United States
}

\author{
Min Huang ${ }^{1}$ (D) Yanyan Lou $^{2} \cdot$ James Pellissier $^{1} \cdot$ Thomas Burke $^{1} \cdot$

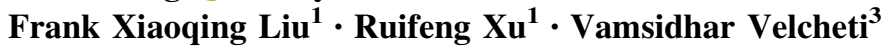

Published online: 15 June 2017

(c) The Author(s) 2017. This article is an open access publication

\begin{abstract}
Objectives Our objectives were to evaluate the cost effectiveness of pembrolizumab compared with standardof-care (SoC) platinum-based chemotherapy as first-line treatment in patients with metastatic non-small-cell lung cancer (NSCLC) that expresses high levels of programmed death ligand-1 (PD-L1) [tumour proportion score (TPS)
\end{abstract}

Electronic supplementary material The online version of this article (doi:10.1007/s40273-017-0527-z) contains supplementary material, which is available to authorized users.

\footnotetext{
Min Huang

Min_Huang@merck.com

Yanyan Lou

Lou.Yanyan@mayo.edu

James Pellissier

James_Pellissier@merck.com

Thomas Burke

Thomas_Burke2@merck.com

Frank Xiaoqing Liu

Xiaoqing.Liu@merck.com

Ruifeng Xu

Ruifeng_Xu@merck.com

Vamsidhar Velcheti

velchev@ccf.org

1 Centre for Observational and Real-world Evidence, Merck \& Co., Inc., 2000 Galloping Hill Road, Kenilworth, NJ 07033, USA

2 Department of Hematology and Oncology, Mayo Clinic, 4500 San Pablo Road, Jacksonville, FL 27710, USA

3 Solid Tumor Oncology, Cleveland Clinic, 9500 Euclid Ave, Mail Code R35, Cleveland, OH 44195, USA
}

$\geq 50 \%$ ], from a US third-party public healthcare payer perspective.

Methods We conducted a partitioned-survival model with a cycle length of 1 week and a base-case time horizon of 20 years. Parametric models were fitted to Kaplan-Meier estimates of time on treatment, progression-free survival and overall survival from the KEYNOTE-024 randomized clinical trial (patients aged $\geq 18$ years with stage IV NSCLC, TPS $\geq 50 \%$, without epidermal growth factor receptor $(E G F R)$-activating mutations or anaplastic lymphoma kinase $(A L K)$ translocations who received no prior systemic chemotherapy) and validated with long-term registry data. Quality-adjusted life-years (QALYs) were calculated based on EuroQoL-5 Dimensions (EQ-5D) utility data collected in the trial. Costs (\$US, year 2016 values) for drug acquisition/administration, adverse events and clinical management were included. Costs and outcomes were discounted at $3 \%$ per year. A series of deterministic and probabilistic sensitivity analyses were performed to test the robustness of the results.

Results In the base-case scenario, pembrolizumab resulted in an expected gain of 1.31 life-years (LYs) and 1.05 QALYs and an incremental cost of \$US102,439 compared with SoC. The incremental cost per QALY gain was \$US97,621/QALY and the incremental cost per LY gain was $\$$ US78,344/LY.

Conclusions Pembrolizumab is projected to be a cost-effective option compared with SoC platinum-based chemotherapy as first-line treatment in adults with metastatic NSCLC expressing high levels of PD-L1. 


\section{Key Points for Decision Makers}

The introduction of new oncology therapies improves survival but can exert financial pressure on US healthcare payers.

This is the first analysis evaluating the cost effectiveness of pembrolizumab compared with standard-of-care treatment in first-line metastatic non-small-cell lung cancer (NSCLC) expressing high levels of programmed death ligand-1 (PD-L1).

Pembrolizumab increased expected quality-adjusted life-years in patients with metastatic NSCLC and is projected to be cost effective from a US third-party public healthcare payer perspective compared with standard-of-care treatment at recently published thresholds of cost effectiveness.

\section{Introduction}

Lung cancer is the most common malignancy worldwide and the most common cause of death from cancer, estimated to be responsible for nearly one in five cancer-related deaths [1]. Non-small-cell lung cancer (NSCLC) represents $85 \%$ of all lung cancers [2]. Long-term prognosis for these patients is poor, since the majority present with advanced disease [3], and half of the patients initially treated for early-stage NSCLC subsequently relapse [4].

Immunotherapy is a new paradigm for the treatment of NSCLC. Pembrolizumab is a humanized monoclonal programmed cell death-1 (PD-1) antibody that inhibits the interaction between PD-1 and programmed death ligand 1 (PD-L1)/PD-L2 receptors. Pembrolizumab received approval from the US FDA in October 2016 [5] for the treatment of metastatic NSCLC whose tumors have high PD-L1 expression [tumor proportion score (TPS) $\geq 50 \%$ ] as determined by an FDA-approved test, with no epidermal growth factor receptor $(E G F R)$ or anaplastic lymphoma kinase $(A L K)$ genomic tumor aberrations, and no prior systemic chemotherapy treatment for metastatic NSCLC.

The KEYNOTE-024 trial demonstrated that patients with metastatic NSCLC with PD-L1 TPS $\geq 50 \%$ treated with pembrolizumab $200 \mathrm{mg}$ every 3 weeks had superior overall survival (OS) compared with patients who received standard-of-care (SoC) treatment, with a hazard ratio (HR) for mortality of $0.60 \quad[95 \%$ confidence interval (CI) $0.41-0.89 ; p=0.005$ ] [5]. Progression-free survival (PFS) was significantly longer with pembrolizumab than with SoC (HR 0.50, 95\% CI 0.37-0.68; $p<0.001$ ). Grade $3-5$ treatment-related adverse events (AEs) were less common with pembrolizumab than with SoC.

The introduction of new oncology therapies can result in financial pressure for US healthcare payers. This is the first economic evaluation of pembrolizumab in first-line NSCLC and is designed to inform value-based reimbursement decisions for payers. The analysis used KEYNOTE-024 data to evaluate the cost effectiveness of pembrolizumab compared with SoC chemotherapy as firstline treatment for metastatic NSCLC expressing high levels of PD-L1 from a US third-party public healthcare payer perspective.

\section{Methods}

A partitioned-survival model [6] was developed to project expected costs and outcomes for pembrolizumab compared with SoC. The main outcome measure was the incremental cost-effectiveness ratio (ICER), expressed as cost per quality-adjusted life-year (QALY) gained; the incremental cost per life-year (LY) gained was also determined. The model was developed in Microsoft ${ }^{\circledR}$ Excel 2010 (Redmond, WA, USA).

Efficacy, safety and utility data were derived from KEYNOTE-024, with a cut-off date of 9 May 2016. This phase III randomized controlled trial compared pembrolizumab with the investigator's choice of five platinumbased regimens in first-line metastatic NSCLC.

\subsection{Target Population}

The target population in the model was based on the KEYNOTE-024 trial population: patients aged $\geq 18$ years (mean age 64 years); diagnosis of stage IV NSCLC without $E G F R$-activating mutations or $A L K$ translocations; no prior systemic chemotherapy treatment for metastatic NSCLC; PD-L1 TPS $\geq 50 \%$.

\subsection{Interventions}

Patients in KEYNOTE-024 were randomized to pembrolizumab $200 \mathrm{mg}$ once every 3 weeks for up to 35 cycles or platinum-based chemotherapy for 4-6 cycles. Nonsquamous patients in the $\mathrm{SoC}$ arm without progressive disease after completion of chemotherapy were eligible to receive pemetrexed maintenance. The SoC regimens included pemetrexed + carboplatin, pemetrexed + cisplatin, gemcitabine + cisplatin, gemcitabine + carboplatin, and paclitaxel + carboplatin (Electronic Supplementary Material [ESM] 1) [7].

Treatment was continued for the specified number of cycles or until disease progression, intolerable toxicity, 
patient withdrawal, or investigator decision. PFS was assessed according to RECIST (Response Evaluation Criteria in Solid Tumors), version 1.1 [8] by means of blinded, independent, central radiologic review (BICR). Patients receiving SoC with verified progressive disease could switch to receive pembrolizumab [7]. Patients were eligible to switch if they had documented progression, did not stop chemotherapy for any other reason than progressive disease, had an Eastern Cooperative Oncology Group (ECOG) score of 0 or 1 at time of progression and had at least 30 days of survival after SOC treatment. Clinically stable patients considered to be deriving clinical benefit by the investigator were eligible to continue pembrolizumab beyond progression. Patients treated with pembrolizumab were assumed to be treated for a maximum of 2 years as per trial protocol and FDA-approved label [9].

\subsection{Model Structure}

The model evaluated the cost effectiveness of pembrolizumab compared with SoC as first-line treatment in the target population (ESM 2). A partitioned-survival model was constructed with three mutually exclusive health states, progression-free (starting state), progressive disease, and death (Fig. 1). Outcomes and costs of AEs, toxicity grade 3-5, were incorporated and weighted by their observed incidence. The time in each health state was used to estimate cumulative total costs and health outcomes over the time horizon for a cohort of patients receiving each intervention.

\subsection{Time Horizon, Discounting, and Perspective}

A time horizon long enough to accommodate patients' lifetime (20 years for base case) was selected to ensure

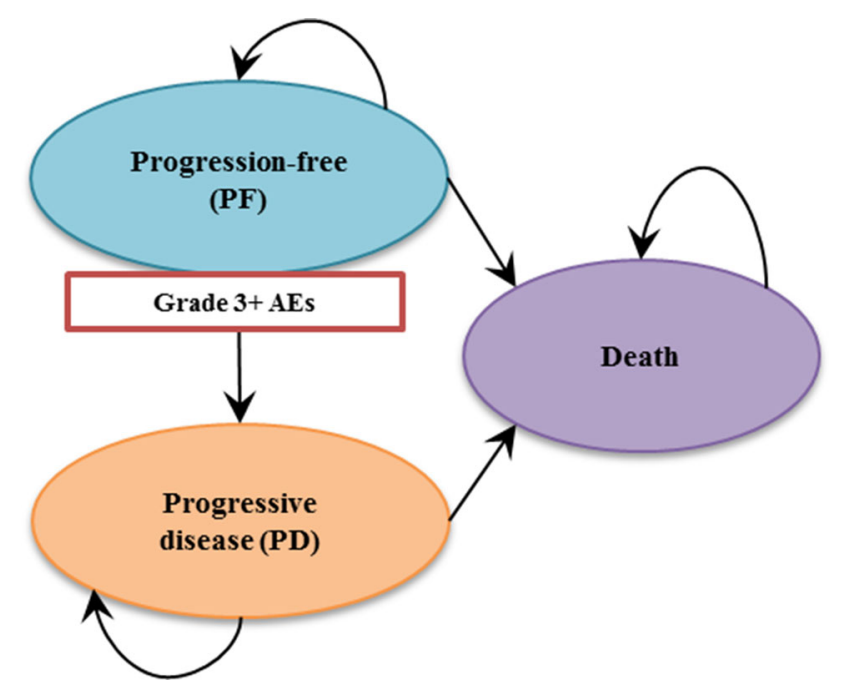

Fig. 1 Model states and transitions. AEs adverse events important differences in costs and outcomes between the interventions were considered. Scenario analyses explored the effect of shorter time horizons ( 5 and 10 years), in view of the short life expectancy of patients with metastatic NSCLC. Costs and outcomes were discounted at $3 \%$ per year, with rates of 0 and $5 \%$ explored in scenario analysis [10].

The analysis was conducted from the perspective of a US third-party public healthcare payer.

\subsection{Clinical Parameters}

The model effectiveness parameters were estimated from KEYNOTE-024 patient-level data for time-on-treatment (ToT), PFS and OS.

Parametric functions were fitted to the Kaplan-Meier (KM) ToT, PFS and OS data, in line with published guidelines, to extrapolate from the trial duration (median follow-up 11.2 months; range 6.3-19.7 months) to the 20-year time horizon. The survival curve fitting was carried out in line with UK National Institute for Health and Care Excellence (NICE) Decision Support Unit (DSU) guidelines [11]. The parametric models fit were the Weibull, the exponential, the log-normal, the log-logistic and the Gompertz distributions. Statistical tests based on the Akaike information criterion (AIC) and the Bayesian information criterion (BIC), combined with visual inspection (comparing fitted distributions to study KM plots), were used to select the best-fitting parametric distributions for the base case. Finally, the clinical plausibility of the extrapolated results was considered in selecting the final distribution functions for the model. Alternative parametric functions were tested in scenario analyses.

\subsubsection{Time-on-Treatment}

ToT data from the KEYNOTE-024 trial were analysed to determine treatment duration and cost, since patients deriving clinical benefit could continue pembrolizumab beyond progression. Parametric functions were fit to the KM ToT data. The AIC and BIC combined with visual inspection were used to select the Weibull distribution for pembrolizumab and the generalized gamma distribution for SoC as the base case. The base-case ToT curves are presented in Fig. 2. Alternative parametric functions and the base-case curves with 20-year time horizon are presented in ESM 2.

\subsubsection{Progression-Free Survival}

For PFS, the KM probabilities from KEYNOTE-024 were used directly up to Week 9, with parametric functions fitted thereafter. This was because the first radiologic tumour response assessment was conducted at Week 9, resulting in 
Fig. 2 Modelled ToT from KEYNOTE-024 for pembrolizumab and standardof-care arms. ToT time on treatment, $S o C$ standard-of-care

Fig. 3 Modelled progressionfree survival from KEYNOTE024 for pembrolizumab and standard-of-care arms. BICR blinded independent central review, ITT intention-to-treat, $P F S$ progression-free survival, $\mathrm{SoC}$ standard of care
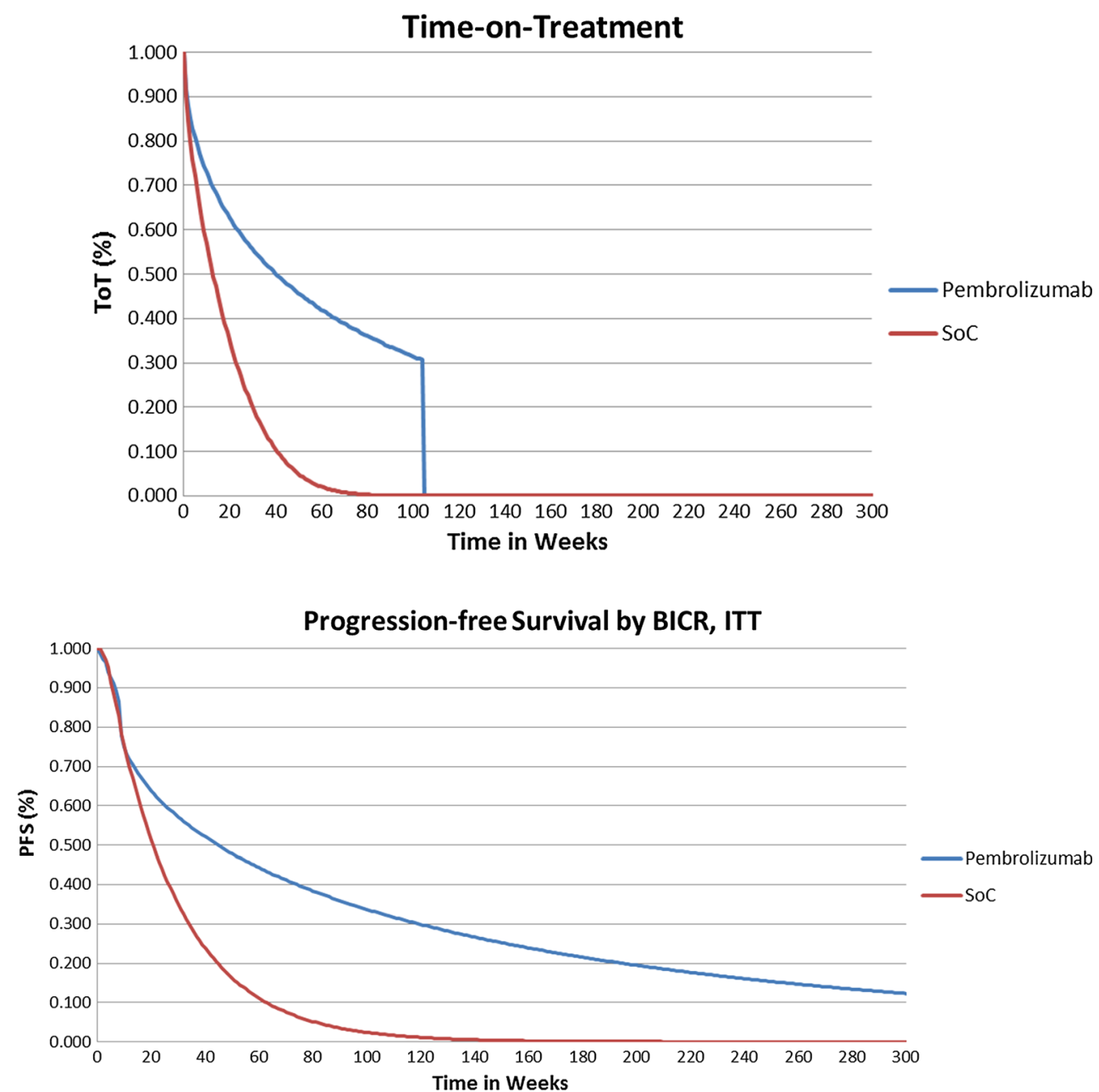

a protocol-driven drop in PFS at this point. The Weibull distribution was the best fit for pembrolizumab, and the exponential parametric function was the best fit for SoC. The base-case PFS curves are presented in Fig. 3. Alternative parametric functions and the base-case curves with 20-year time horizon are presented in ESM 3.

\subsubsection{Overall Survival}

In KEYNOTE-024, 43.71\% of patients from the SoC group crossed over to receive pembrolizumab after disease progression, which was anticipated to diminish the survival benefit of pembrolizumab. A simplified two-stage approach as described by Latimer and Abrams [12] and Latimer [13] was implemented to perform an OS analysis adjusting for switching in the SoC arm. Through this approach, OS treatment effect estimate was adjusted to correct for the bias induced by treatment switch.

Two datasets are available for use in the OS analysis: (1) the intention-to-treat (ITT) dataset included patients who switched from SoC to pembrolizumab following disease progression, and (2) a 'switching-adjusted' dataset used the two-stage adjustment method to adjust the survival benefit of patients who switched to pembrolizumab,

The ITT dataset was used in the base-case analysis, as it better represented the real-world situation given the existing FDA approval of pembrolizumab as second-line therapy for metastatic NSCLC expressing PD-L1 [9]. The OS impact of subsequent therapies was assumed to be reflected within the OS KM data from KEYNOTE-024. The switching-adjusted dataset was explored in a scenario analysis.

For the OS outcomes, the standard parametric curves tested did not provide a good visual fit to the observed KM data, so a two-phase piecewise model was applied. Cut points were determined by estimating structural changes to the KM curve using Chow tests $[14,15]$. The area under the KM curve was used directly for the first phase until the cut-off, and an exponential distribution was used to estimate OS for up to 5 years of follow-up. The cut-off point identified by the Chow test was Week 32 for pembrolizumab, Week 38 for SoC (without adjustment), and Week 25 for SoC with adjustment. A constant hazard rate, derived from long-term external data for patients with stage IV NSCLC from the Surveillance, Epidemiology and End 
Fig. 4 Modelled overall survival with the hazard rate from SEER data applied after year 5. a intention-to-treat analysis without switching adjustment; b switching adjusted. $K M$ Kaplan-Meier, $O S$ overall survival, SEER Surveillance, Epidemiology, and End Results Program, SoC standard of care
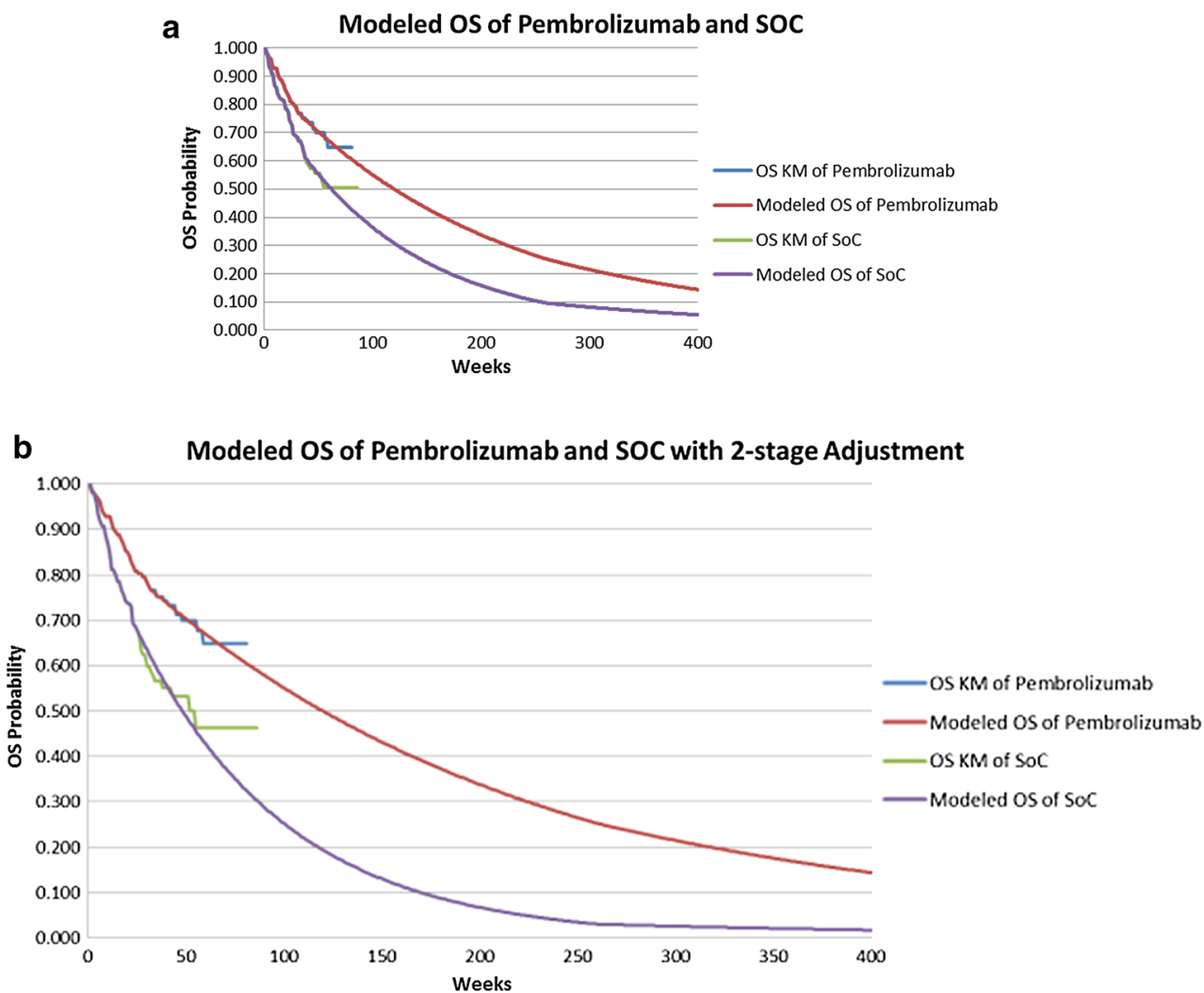

Results (SEER) database, was applied to the OS model after Year 5 [16].

The base-case OS curves are presented in Fig. 4. Alternative parametric functions and the base-case curves with 20-year time horizon are presented in ESM 4.

\subsubsection{Subsequent Therapies}

The percentage of patients receiving subsequent therapy $(45.51 \%)$ was estimated from the Flatiron database [17]. Flatiron is a nationally representative electronic health record data that includes 162 practices from different regions in the USA. We included patients (aged $\geq 18$ years) newly diagnosed with metastatic NSCLC initiating firstline anticancer therapy (November 2012-January 2015, with follow-up through July 2015). In the pembrolizumab arm, and SoC arm with switching adjustment, all such patients were assumed to receive chemotherapy. In the SoC arm without switching adjustment, $43.71 \%$ of patients were assumed to receive pembrolizumab and $1.80 \%$ were assumed to receive chemotherapy based on the switching rate observed from the KEYNOTE-024 data.

\subsubsection{Adverse Events}

The model included all-cause AEs of grade 3 or higher reported in $\geq 5 \%$ of patients in KEYNOTE-024 for either arm. Pneumonitis, an immune-mediated AE with a high management cost, was included based on feedback from clinicians received in an advisory panel review of the model. Model input data for each AE are shown in Table 1.

\subsubsection{Utility Data}

The utility data used in the base case (Table 1) were based on EuroQol-5 Dimensions, 3 Levels (EQ-5D-3L) data collected in KEYNOTE-024. The EQ-5D questionnaire was administered at treatment cycle 1, 2, 3, 6, 9, 12 and every third cycle as long as patients were on study treatment, as well as at the treatment discontinuation visit and at a 30-day post-treatment safety follow-up visit. The generic health statuses assessed from the EQ-5D questionnaire were converted to population-based utility values using published algorithms. For the base-case analysis, US-based scores [18] were applied to US patients, UK-based scores [19] for UK patients and EU-based scores [20] for all other patients. Two approaches of defining health state utilities were considered: time-to-death and progression-based health states. The time-to-death approach, recently described by Hatswell et al. [21], reflects the decline in cancer patients' quality of life as they approach death. Four timeto-death categories (over 360, 180-360, 30-180 days, and under 30 days) were examined for use in the model base case. Pooled data were used as there were no significant 
Table 1 Key input data

\begin{tabular}{|c|c|c|c|c|c|}
\hline $\begin{array}{l}\text { Modelling fit and } \\
\text { extrapolation } \\
\text { approach }\end{array}$ & Overall survival & \multicolumn{2}{|l|}{ PFS } & \multicolumn{2}{|c|}{ Time-on-treatment } \\
\hline PEM & $\begin{array}{l}\text { KEYNOTE-024 KM to } 32 \text { weeks, } \\
\text { exponential to } 5 \text { years, SEER data } \\
\text { thereafter }\end{array}$ & \multicolumn{2}{|c|}{$\begin{array}{l}\text { KEYNOTE- } 024 \text { KM to } \\
9 \text { weeks, Weibull } \\
\text { thereafter }\end{array}$} & \multicolumn{2}{|c|}{$\begin{array}{l}\text { Weibull to } 2 \text { years (maximum } 2 \text { years of } \\
\text { treatment assumed) }\end{array}$} \\
\hline SoC & $\begin{array}{l}\text { KEYNOTE-024 KM to } 38 \text { weeks, } \\
\text { exponential to } 5 \text { years, SEER data } \\
\text { thereafter }\end{array}$ & \multicolumn{2}{|c|}{$\begin{array}{l}\text { KEYNOTE- } 024 \mathrm{KM} \text { to } \\
9 \text { weeks, exponential } \\
\text { thereafter }\end{array}$} & \multicolumn{2}{|c|}{$\begin{array}{l}\text { Generalized gamma ( } 4-6 \text { cycles of platinum, } \\
\text { no treatment cap for pemetrexed } \\
\text { maintenance) }\end{array}$} \\
\hline \multicolumn{4}{|c|}{ Time-to-death utilities (pooled treatment groups from KEYNOTE-024) } & $n^{\mathrm{a}}$ & Utilities (95\% CI) \\
\hline \multicolumn{6}{|c|}{ Time to death (days) } \\
\hline$\geq 360^{\mathrm{b}}$ & & & & 54 & $0.805(0.767-0.843)$ \\
\hline$(180,360)$ & & & & 26 & $0.726(0.684-0.767)$ \\
\hline$(30,180)$ & & & & 68 & $0.632(0.592-0.672)$ \\
\hline$<30$ & & & & 21 & $0.537(0.425-0.650)$ \\
\hline \multicolumn{2}{|c|}{ Costs (proportion paid by third-party payers ${ }^{\mathrm{c}}$ ) } & Cost (\$US) & \multicolumn{3}{|l|}{ Source } \\
\hline \multicolumn{2}{|c|}{ Administration cost for first hour CTX infusion } & 224 & \multicolumn{3}{|c|}{ CPT: 96413 [23] } \\
\hline \multicolumn{2}{|c|}{ Administration cost for additional hour CTX infusion } & 34 & \multicolumn{3}{|c|}{ СРT: 96415 [23] } \\
\hline \multicolumn{2}{|c|}{ Administration cost per hour for subsequent CTX infusion } & 34 & \multicolumn{3}{|c|}{ CPT: 96417 [23] } \\
\hline \multicolumn{2}{|c|}{ PEM weekly cost of disease management in PF } & 772 & \multicolumn{3}{|c|}{ Estimated from healthcare resource use in KEYNOTE-024 } \\
\hline \multicolumn{2}{|c|}{ SoC weekly cost of disease management in $\mathrm{PF}$} & 1158 & \multicolumn{3}{|c|}{ Estimated from healthcare resource use in KEYNOTE-024 } \\
\hline \multicolumn{2}{|c|}{ Weekly cost of disease management in PD } & 1791 & \multicolumn{3}{|c|}{ Ramsey et al. [29] } \\
\hline \multicolumn{2}{|c|}{ PEM post-discontinuation therapy cost } & 11,084 & \multicolumn{3}{|c|}{ Ramsey et al. [29] } \\
\hline \multicolumn{2}{|c|}{ SoC post-discontinuation therapy cost } & 27,989 & \multicolumn{3}{|c|}{ KN024 and Ramsey et al. [29] } \\
\hline \multicolumn{2}{|c|}{ Cost of terminal care } & 31,114 & \multicolumn{3}{|c|}{ Chastek et al. [34] } \\
\hline
\end{tabular}

Costs and incidence of relevant adverse events (grade $3+$ )

\begin{tabular}{|c|c|c|c|c|c|}
\hline \multirow[t]{2}{*}{ Adverse event } & \multicolumn{2}{|c|}{ Incidence (\%) (KEYNOTE-024) } & \multirow[t]{2}{*}{$\%$ hospitalized $^{\mathrm{d}}$} & \multirow{2}{*}{$\begin{array}{l}\text { CMS hospitalization cost } \\
\text { per event [38] (\$US) }\end{array}$} & \multirow[t]{2}{*}{ Sources (DRG code) [38] } \\
\hline & PEM & SoC & & & \\
\hline Anaemia & 4.5 & 23.3 & 50 & 7969.56 & $808 ; 809 ; 810$ \\
\hline $\begin{array}{l}\text { Neutropenia or neutrophil } \\
\text { count decreased }\end{array}$ & 0.0 & 18.0 & 50 & 7969.56 & $808 ; 809 ; 810$ \\
\hline Pneumonia & 1.9 & 7.3 & 100 & 5963.80 & $193 ; 194 ; 195$ \\
\hline $\begin{array}{l}\text { Thrombocytopenia or platelet } \\
\text { count decreased }\end{array}$ & 0.0 & 12.0 & 30 & 5308.86 & 951 \\
\hline Pneumonitis & 2.6 & 0.7 & 100 & 8766.53 & $177 ; 178 ; 179$ \\
\hline
\end{tabular}

$C I$ confidence interval, $C P T$ current procedural terminology, $C T X$ chemotherapy, $D R G$ diagnosis-related group, $K M$ Kaplan-Meier, $P D$ progressive disease, $P D-1$ programmed-cell death receptor $1, P E M$ pembrolizumab, $P F$ progression-free, $P F S$ progression-free survival, $S E E R$ Surveillance, Epidemiology and End Results, $S o C$ standard-of-care

${ }^{a}$ Number of patients with non-missing EQ-5D index score

b This time-to-death category includes the records of patients whose death dates were observed or censored $\geq 360$ days after the report of EQ-5D scores. Other categories only include the records of patients with an observed death date

c $80 \%$ of the total costs were assumed to be covered by healthcare payers

${ }^{\mathrm{d}}$ Based on discussion with clinical experts in an advisory panel meeting 
differences in utility values in each time-to-death category between the treatment arms [22].

\subsection{Resource Utilization and Cost Inputs}

The cost inputs considered in the cost-effectiveness analysis were regimen-related costs, including drug acquisition, administration and pre-medication costs, disease management costs, subsequent therapy costs, terminal care costs and AE management costs (summarized in Table 1). It was assumed that $80 \%$ of healthcare costs would be paid by public healthcare payers [23]. All costs were updated to \$US, year 2016 values using the Medical Care Consumer Price Index [24].

\subsubsection{Regimen-Related Costs}

Pembrolizumab is available in single-use vials of $100 \mathrm{mg}$ and is administered at a dose of $200 \mathrm{mg}$ every 3 weeks. The list price for pembrolizumab is $\$$ US4381 per 100-mg vial, therefore the cost per dose is \$US8762 [25]. Dosage of SoC chemotherapy regimens is based on patients' body surface area. The average number of vials per dose of each regimen was calculated using the body surface area distribution [mean $1.84 \mathrm{~m}^{2}$, standard deviation (SD) $0.26 \mathrm{~m}^{2}$ ] of patients in the Flatiron database [17]. The cost of SoC was \$US3472 per dose, based on the distribution of each regimen in KEYNOTE-024 (ESM 1). The cost per dose of pemetrexed maintenance was estimated at \$US4907. Drug administration costs for intravenous infusions based on the year 2016 Center for Medicare and Medicaid Services (CMS) payment rates [23] are shown in Table 1. The number of treatment cycles in the model was based on the estimated ToT. Treatment duration was modelled per KEYNOTE-024 and FDA-approved label up to 4-6 cycles for platinum and up to 2 years for pembrolizumab [9].

Costs of anti-emetic prophylaxis were identified from National Comprehensive Cancer Network (NCCN) guidelines and estimated at \$US286.55 and \$US5.95 per cycle for cisplatin or carboplatin, respectively [26].

\subsubsection{Disease Management Costs}

For the progression-free state, disease management costs were based on healthcare resource utilization (HCRU) data observed in KEYNOTE-024. The HCRU data captured non-study-related healthcare visits (including visits for home-based hospice, home healthcare, non-study laboratory, radiation therapy, nurse, primary care, and medical specialists), hospitalization and emergency room visits. Pembrolizumab was associated with lower HCRUs than SoC. The progression-free state HCRU in the KEYNOTE024 was described in detail in Huang et al. [27]. The unit costs were based on 2016 CMS payment rates [28]. To inform disease management costs for the progressive disease state, a literature review was conducted, which identified published studies whose estimates were based on US commercial claims [29, 30] and SEER Medicare [31-33]. However, the SEER-Medicare studies over-estimated disease management costs as defined for the model, as intravenous anti-cancer therapy costs were not excluded. Thus, the model base-case value (Table 1) is based on reported results that used commercial claims [29] from initiation of second-line intravenous anti-cancer therapy after excluding intravenous anti-cancer therapy costs. Sensitivity analyses of this parameter embrace the range of reported values from the literature [29, 30]. Disease management costs in the post-progression health state were estimated based on cost data reported by Ramsey et al. [29]. Disease management costs were estimated by evaluating the 1704 patients with NSCLC, and included the following cost categories: (1) inpatient, (2) emergency department, (3) ambulatory (including chemotherapy administration costs in office or outpatient setting), (4) other medical (captured laboratory costs, including the actual processing of the specimen), (5) retail pharmacy. We excluded the cost of 'chemotherapy administration' as a disease management cost, as it was included separately in the model within subsequent therapy costs.

\subsubsection{Subsequent Therapy Costs}

To estimate the post-discontinuation therapy costs for SoC patients, the weighted average of subsequent treatment weighted by the rate of switching to pembrolizumab was implemented. Average treatment duration with pembrolizumab was assumed to be 183 days based on the treatment-switching patients in KEYNOTE-024. Patients receiving pembrolizumab were assigned second-line chemotherapy post-progression. The average cost of second-line chemotherapy was estimated to be $\$$ US24,358 per patient based on the cost of chemotherapy administrations in a US healthcare claim database [29].

\subsubsection{Terminal Care Costs}

A literature review was conducted to inform the cost of terminal care in the final 30 days of life. Five studies were identified that reported direct medical costs in the end-of life period [30, 34-37]. All studies were limited to patients with lung cancer, with the exception of Chastek et al. [34], who included all cancer patients with analyses stratified by primary cancer site. The studies used varied sources, including SEER-Medicare [30], integrated health systems databases $[35,36]$ and commercial administrative claims $[34,37]$. Costs in these studies were reported over 
a variety of intervals, including the period of time from last chemotherapy dose to death [30], the time from initial disease progression to death [35] and the last 6 months of life [34, 36, 37]. Chastek et al. [34] was the only study to report costs within the terminal period by 30-day increments, showing that direct medical costs increased during the last 30 days of life because of increases in hospitalization and hospice costs; these were used for the model base case. Importantly, the costs in the last 30-day period (\$US31,114) were higher than the average monthly cost over the 6-month period (\$US14,748). Chastek et al. [34] did not report resource utilization, but their reported cost estimates reflected the sum of health plan and patient paid amounts, by cancer-related cost category. These categories included (1) inpatient, (2) hospice (inpatient and outpatient), (3) chemotherapy, (4) growth factors [erythropoiesis-stimulating agents (ESAs) and granulocyte colony-stimulating factors (GCSFs)], (5) radiation, (6) emergency room visits, (7) office visits, (8) hospital outpatient procedures and (9) other outpatient cancer-related services.

\subsubsection{Adverse Event Management Costs}

Incidence and costs of selected grade 3 or higher AEs are summarized in Table 1. Hospitalization costs related to AE management were considered in the model. The rates of hospitalization were provided by clinical experts. The hospitalization costs for managing each $\mathrm{AE}$ were obtained from the 2016 CMS payment rates [38]. Based on incidences of AEs and management costs, the total average cost per patient for managing AEs was estimated to be \$US2334 for pembrolizumab and \$US521 for SoC.

\subsection{Sensitivity Analyses}

One-way deterministic sensitivity analyses (DSAs) were conducted to evaluate the sensitivity of the model to plausible changes to each key model parameter. A probabilistic sensitivity analysis (PSA) was conducted to test the robustness of the model with respect to uncertainty in model input parameters. In this analysis, each key parameter was assigned a theoretical probability distribution. Correlated parameters were assigned a joint distribution. For example, the two parameters in the Weibull function fitted to ToT were assigned a multivariate-normal distribution. The PSA was performed using a second-order Monte Carlo simulation with 1000 iterations. DSA ranges and parameter distributions used in the PSA are detailed in ESM 6.

Multiple scenario-based sensitivity analyses were also performed. One such analysis explored the impact of differing practice patterns implied by selection of alternative
OS data. In particular, analyses representing settings where PD-1 inhibitors are unavailable as second-line or later treatment were explored using a two-stage adjustment of the treatment OS that removed the effect of switching treatment from chemotherapy to pembrolizumab. Additional scenario-based sensitivity analyses adjusted the model time horizon, the discounting of costs and outcomes, and the impact of PD-L1 testing costs, as well as the application of alternative plausible parametric functions to the extrapolation of OS, PFS and ToT.

\subsection{Model Validation}

The modelling process followed good practice. Health economists, clinicians and pharmacy professionals who were experts in oncology economics evaluated this model and other analogous versions. In addition to these singular evaluations, an expert panel consisting of three medical oncologists, one oncology pharmacist and one biostatistician provided detailed review and feedback. Evaluation covered not only the conceptual model but also the model data, assumptions, methods, analyses and results. The panel feedback was used to provide insight into what AEs were important for inclusion in the model, It also provided verification to the modelling approach, analysis plans, clinical plausibility of clinical inputs (e.g. hospitalization rates for AEs), duration of treatment effects and modelled outcomes such as projections of OS and PFS. Where consensus was not achieved, the panel provided guidance on the scope of sensitivity analyses. The modelling process, conceptual model and the computerized model were evaluated against and satisfied the published AdViSHE criteria for assessment of the validation status of healtheconomic decision models [39].

The OS extrapolation was validated with external observation data. The clinical plausibility of the projected long-term outcomes was also verified by independent clinical experts. The projected OS curve of SoC was compared with the survival data collected from the Flatiron Health database [17] in patients aged $\geq 18$ years newly diagnosed with metastatic NSCLC initiating first-line anticancer therapy (November 2012-January 2015, with follow-up through July 2015). As shown in ESM 5, the OS curve from the Flatiron data matched closely with the projected OS curve of SoC with switching adjustment. The model-projected 5-year survival with $\mathrm{SoC}$ with switching adjustment was $3.1 \%$, which is close to the 5-year survival rate of $3.21 \%$ estimated from the SEER data [16] for metastatic NSCLC.

No observational data are currently available to validate the OS extrapolation of first-line pembrolizumab in NSCLC as this is a newly approved indication. The plausibility of the approach to modelling OS was supported by 
Table 2 Base-case results

\begin{tabular}{llll}
\hline & SoC & PEM & Incremental PEM vs. SoC \\
\hline Life-years & 2.05 & 3.35 & 1.31 \\
Expected time in progression free state (years) & 0.55 & 2.16 & 1.62 \\
Expected time in progressive state (years) & 1.50 & 1.19 & -0.31 \\
QALYs & 1.55 & 2.60 & 1.05 \\
Costs (\$US) & 260,223 & 362,662 & 102,439 \\
Drug acquisition cost & 25,090 & 120,478 & 95,388 \\
Pre-medication cost & 328 & 0 & -328 \\
Drug administration cost & 1683 & 3854 & 2171 \\
Disease management cost & 173,227 & 198,458 & 25,231 \\
Post-discontinuation therapy cost & 27,989 & 11,084 & $-16,905$ \\
Terminal care cost & 29,573 & 28,268 & -1305 \\
Adverse event cost & 2334 & 521 & -1813 \\
Incremental cost-effectiveness ratio (\$US) & & & \\
Cost per life-year gained & & & 78,344 \\
Cost per QALY gained & & & 97,621 \\
\hline
\end{tabular}

$P E M$ pembrolizumab, $Q A L Y$ quality-adjusted life-year, $S o C$ standard-of-care

feedback from clinician advisory panel review of the projected treatment effects of pembrolizumab and 5-year and 10 -year survival percentages.

\section{Results}

\subsection{Base-Case Results}

Base-case results (Table 2) projected that pembrolizumab would result in a longer expected time in the progressionfree state (1.62 years), more LYs (1.31) and QALYs (1.05), and an incremental cost of \$US102,439 compared with SoC over the 20-year time horizon. Pembrolizumab was also projected to be associated with higher overall costs, driven by drug acquisition costs. The incremental cost per QALY gained with pembrolizumab over SoC was \$US97,621, and the incremental cost per LY gained was \$US78,344 (Table 2).

\subsection{Scenario Analyses}

In the scenario with OS adjusted for patients who switched from SoC to pembrolizumab in KEYNOTE-024, the number of LYs gained was 1.94 and the number of QALYs gained was 1.55 over the 20-year time horizon, which resulted in an expected ICER of \$US91,658/LY and \$US114,194/QALY for pembrolizumab versus SoC (ESM 7). Using PFS as a proxy for ToT with pembrolizumab resulted in an ICER of $\$$ US83,232/LY and \$US103,711/QALY.

To be treated with pembrolizumab, patients must have a PD-L1 test conducted and the test must show PD-L1 expression in greater than $50 \%$ of tumor cells (TPS $\geq 50 \%$ ). The focus of these analyses has been to evaluate the cost effectiveness of pembrolizumab for such patients who have been identified for treatment. Further examination of the policy of testing all patients for PD-L1 and treating identified patients with pembrolizumab versus a no-testing strategy with all patients treated with chemotherapy SoC was performed. Pembrolizumab would be expected to result in an incremental cost of $\$$ US98,281/ QALY gained or an incremental cost of \$US78,873/LY gained. Including cost of PD-L1 testing has a very small impact on the model results.

With a 5-year time horizon, the ICER was \$US99,998/ LY and \$US122,024/QALY; with a 10-year time horizon, the ICER was \$US83,065 and \$US103,101/QALY. Changes in the discount rate had little effect on the ICER. With discount rates for costs of $0 \%$ and for outcomes of $5 \%$ (the least favourable combination), the ICER was \$US94,007/ LY and \$US116,884/QALY; with discount rates for costs of $5 \%$ and for outcomes of $0 \%$ (the most favourable combination), the ICER was $\$$ US64,025/LY and \$US80,043/QALY. Applying alternative plausible parametric functions to the extrapolation of OS, PFS and ToT from the trial data to the 20-year model time horizon did not have large effects on the results (ESM 8).

\subsection{Sensitivity Analyses}

The tornado diagram depicted in Fig. 5 shows the impact of parameter variation on the ICER as derived from the one-way DSA. The variables with the most impact on the ICER were extrapolation of PFS and OS, extrapolation of ToT for pembrolizumab and utilities for time $\geq 360$ days 


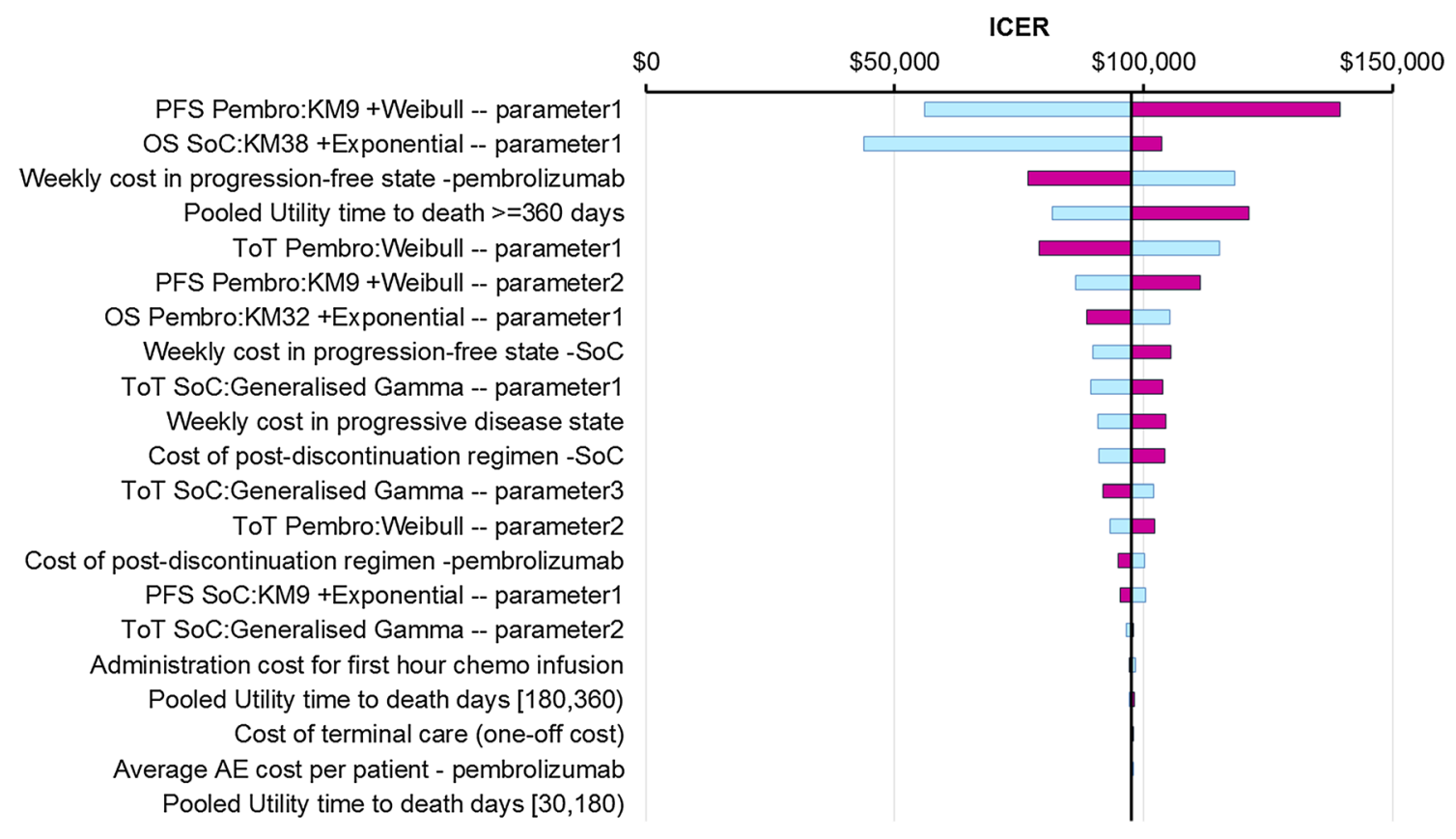

¿ Lower Bound $\square$ Upper Bound

Fig. 5 One-way sensitivity analysis: tornado diagram for the incremental cost-effectiveness ratio (per quality-adjusted life-year) of pembrolizumab vs. standard-of-care. $A E$ adverse event, ICER

from death. Post-discontinuation costs, costs for terminal care, infusion costs and AE management costs had less impact on the analyses.

The PSA resulted in mean expected incremental costs of \$US100,917, mean expected incremental QALYs of 1.01 and a mean expected ICER of \$US99,882/QALY for pembrolizumab compared with SoC. Figure 6a shows the results of each iteration within a scatter plot, and Fig. $6 \mathrm{~b}$ shows the cost-effectiveness acceptability curve. The probability that the ICER would be below $\$$ US50,000/ QALY and \$US100,000/QALY was 15 and 49\%, respectively. The probability that the ICER would be below \$US150,000/QALY was $87 \%$.

\section{Discussion}

The cost effectiveness of pembrolizumab versus docetaxel has already been demonstrated in a second-line setting for patients with NSCLC and a TPS $\geq 50 \%$ [40]. This economic evaluation compared pembrolizumab with SoC platinum-based chemotherapy as first-line treatment for patients with metastatic NSCLC expressing high levels of PD-L1. Base-case results indicated that, compared with SoC over a 20 -year time horizon, pembrolizumab would be expected to result in an additional $1.31 \mathrm{LYs}$ and an additional 1.05 QALYs gained, without adjustment for incremental cost-effectiveness ratio, $K M$ Kaplan-Meier, $O S$ overall survival, $P F S$ progression-free survival, $S o C$ standard of care, ToT time on treatment

treatment switching, at an additional cost of $\$$ US102,439, giving an incremental cost of \$US97,621/QALY gained or an incremental cost of $\$$ US78,344/LY gained. The main driver of the increased cost with pembrolizumab was the drug acquisition cost. Sensitivity analysis indicated the results were most sensitive to the extrapolation of PFS, OS and ToT, and cost in the progression-free state. The analysis was specifically conducted for the population indicated by the pembrolizumab label supported by KEYNOTE-024. The results are not generalizable to lower PD-L1 levels (TPS $<50 \%$ ).

There has been no general agreement on a cost-effectiveness ratio threshold for the USA. Braithwaite et al. [41] cite multiple decision rules, including the commonly cited \$US50,000/QALY gained and the World Health Organization (WHO) criteria [42] of three times the gross domestic product (GDP) per capita per disability-adjusted life-year, which is estimated to equate to $\$$ US171,660 in 2016 [43]. Their analyses suggested a range for the willingness-to-pay threshold (adjusted to \$US, year 2016 values) of $\$$ US122,360-340,000 and \$US140,392-382,536 for LYs gained and QALYs gained, respectively [38]. A more recent analysis by Neumann et al. [44] recommended using an ICER range of between \$US100,000 and \$US150,000/ QALY gained, although analyses should allow for examination of multiple thresholds up to \$US200,000/QALY gained. The Institute of Clinical and Economic Review 
Fig. 6 Probabilistic sensitivity analysis: a cost-effectiveness plane, $\mathbf{b}$ cost-effectiveness acceptability curve. PSA probabilistic sensitivity analysis, QALYs qualityadjusted life-years
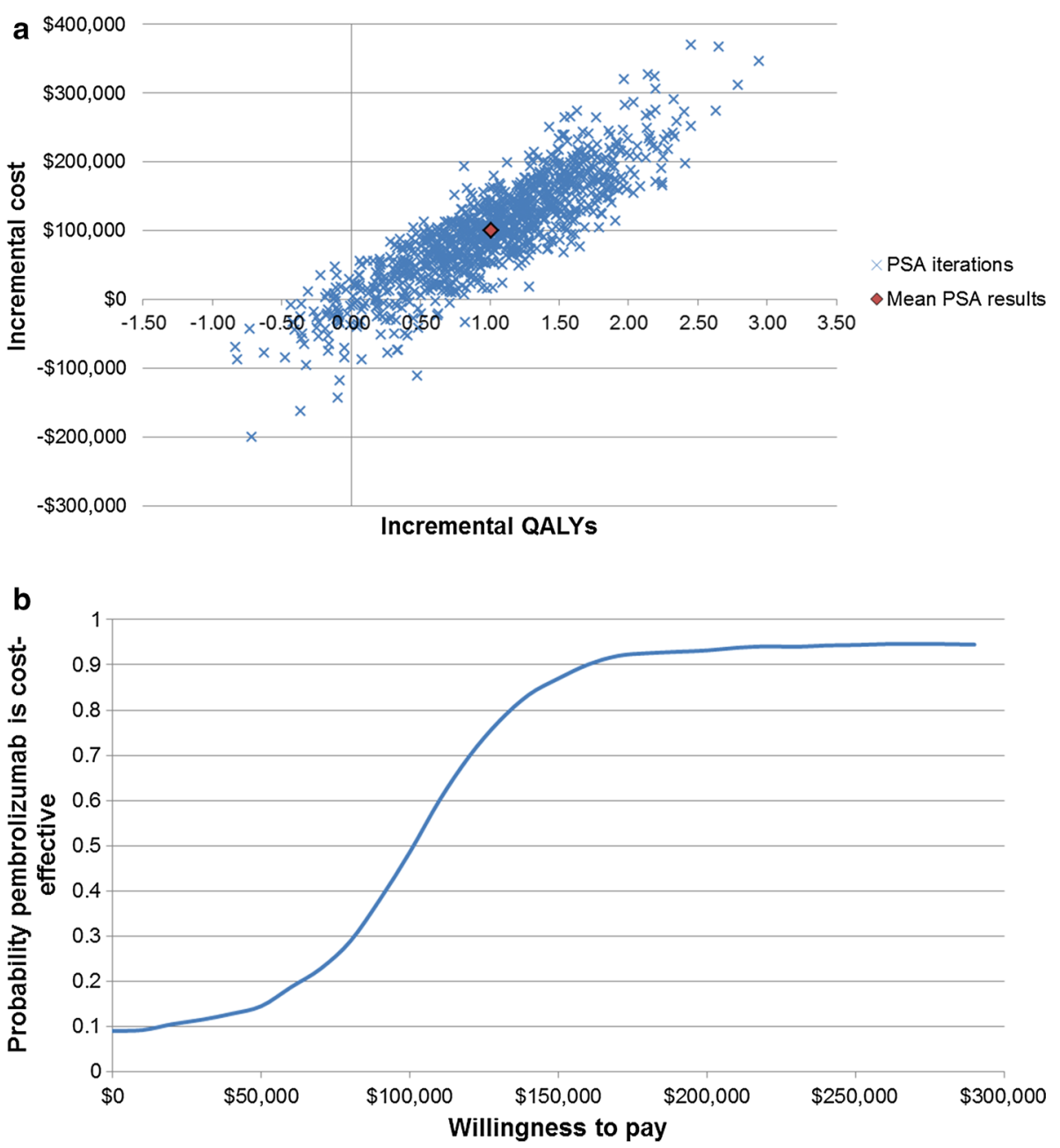

[45] recently made a recommendation of a range of costeffectiveness thresholds between \$US50,000 and \$US150,000 per QALY. The base-case ICER for pembrolizumab of \$US97,621/QALY in the current analysis falls within these ranges of acceptable thresholds. Results from the PSA showed an $87 \%$ probability that the ICER would be below \$US150,000/QALY. Hypothetical modelling and simulated data have suggested the partitionedsurvival model may have some potential for bias [46, 47]. However, an empirical analysis using real-world data from three clinical trials found that the partitioned-survival approach produced ICER estimates similar to those generated by Markov modelling in metastatic NSCLC, indicating that the suggestion of consistent bias is unfounded [48]. Additionally, the two-phase piecewise model for OS extrapolation has been noted as a preferred approach by the NICE Evidence Review Group compared with fitting a parametric function distribution over the entire time horizon $[49,50]$.

The base-case analysis included patients who switched from SoC to pembrolizumab after progression in
KEYNOTE-024. This is consistent with real-world clinical practice, as pembrolizumab is currently indicated in the USA as second-line therapy for metastatic NSCLC expressing PD-L1 [9]. However, such switching has the effect of diluting the survival benefit with pembrolizumab while increasing costs in the $\mathrm{SoC}$ group (as some $\mathrm{SoC}$ patients receive pembrolizumab as second-line treatment). A scenario analysis using OS data adjusted for switching resulted in higher estimates for LYs and QALYs gained with pembrolizumab, with a slightly higher ICER (\$US114,194/QALY), compared with the base case (\$US97,621/QALY).

A key strength of this analysis is its use of efficacy and safety data from a randomized controlled trial directly comparing pembrolizumab and SoC. However, the analysis also has several limitations. Resource use from the clinical trial may not represent that of real-world clinical practice. Like all clinical trials, KEYNOTE-024 was conducted in a selected patient population that met trial inclusion and exclusion criteria, and may not reflect patients seen in real-world clinical practice. Similarly, 
KEYNOTE-024 was primarily conducted in large, urban, academic centres within and outside the USA, where clinical practice may differ from that in community practices and other treatment locations. In KEYNOTE-024, $37.4 \%$ of non-squamous patients received pemetrexed continuation maintenance. This compares with data from the Flatiron database, which found $26.6 \%$ of non-squamous patients receiving induction therapy received continuation maintenance therapy [51]. As the impact on efficacy associated with these different rates is not known, the bias to the ICER results is also unknown. In the SoC arm, $43.7 \%$ of patients had within-trial switching to pembrolizumab and $49.7 \%$ received PD-1 inhibitors, including indirect switching. Estimations from the Flatiron database [17] (in patients diagnosed between 1 October 2015 and 31 August 2016) indicated the percentage of patients who received PD-1 inhibitors as subsequent therapies was $49.0 \%$ in patients with PD-L1 $\geq 50 \%$ metastatic NSCLC who received platinum-based chemotherapy as first-line therapy. The switching rate in the trial was similar to that in real-world evidence.

The costs of grade 3-5 AEs with an incidence rate $<5 \%$ in both treatment groups were not included in the analysis, leading to underestimation of $\mathrm{AE}$ costs. However, the bias is not expected to be large considering the low incidence rate of the excluded AEs. In addition, sensitivity analyses have been conducted to vary the $\mathrm{AE}$ costs by $\pm 50 \%$, and the results indicated this variation in $\mathrm{AE}$ costs only had a small impact on the ICER.

The analyses indicated pembrolizumab was cost effective at its published list price. Any hypothetical discount does not qualitatively change this result. Further sensitivity analyses showed that pembrolizumab would be cost effective under the threshold of \$US100,000/QALY if its cost increased up to $3 \%$, and cost effective under the threshold of \$US150,000/QALY if its cost increased up to $59 \%$.

Evaluation using cost-effectiveness analyses does not guarantee affordability. Understanding the potential budget impact is also necessary. Wu et al. [52] reported a budget impact analysis to evaluate the cost burden associated with pembrolizumab in treating first-line metastatic NSCLC to US payers. It projected the incidence of metastatic NSCLC to be 313 patients for a health plan with 1 million members. Assuming an $80 \%$ PD-L1 testing rate and $30 \%$ prevalence of PD-L1 $\geq 50 \%$ within these patients, 55 patients were projected to be eligible for pembrolizumab treatment. These analyses showed a budget impact of pembrolizumab of \$USO.12 per member per month.

At data cut-off from KEYNOTE-024 for this analysis, the median follow-up in the trial was $<1$ year; therefore, extrapolation was necessary to model a lifetime time horizon. Uncertainty is inherent in this process, and although several survival curve options were explored in scenario analyses, longer-term data will be important to test the model results in future clinical practice.

\section{Conclusion}

Compared with SoC platinum-based chemotherapy, pembrolizumab showed significantly longer OS and PFS and fewer treatment-related AEs in patients with previously untreated metastatic NSCLC with PD-L1 TPS $\geq 50 \%$. The results of this cost-effectiveness analysis suggest that pembrolizumab improves life expectancy and expected quality-adjusted life time and can be considered a costeffective option compared with $\mathrm{SoC}$ in these patients with biomarker-identified metastatic NSCLC from a third-party US public payer perspective.

Data availability statements Several datasets were used for this analysis: (1) efficacy, safety, patient-reported outcomes and healthcare resource utilization data collected in phase III KEYNOTE-024 trial. The patient-level data are not publicly available, but the results of the trials have been presented in several publications. The trial results supporting the findings of this analysis are available within the article and its supplemental material. (2) Observational data from the SEER and Flatiron databases, for which references are provided in the article. (3) Cost data used in the model were obtained from CMS or other publications. (4) The model was developed in Excel and is not publicly available, but is available from the authors upon request, with permission of Merck and Co. Inc. and receipt of a signed confidentiality agreement.

Acknowledgements The authors thank Ebony Samuels and Hui Tan (PAREXEL) for manuscript co-ordination and project management, Carole Nadin (on behalf of PAREXEL) for medical writing assistance and Jane Liao (Merck \& Co. Inc.) and Fansen Kong (Merck \& Co. Inc.) for their dedicated work on data analyses.

Author Contributions MH led the development of this health economic analysis and had full access to all the data in the study. $\mathrm{MH}$ takes responsibility for the integrity of the data and the accuracy of the analysis. All authors contributed to the study design and methodology, interpretation of the results and development and writing of the draft manuscript and approved the final version.

\section{Compliance with Ethical Standards}

Funding This study and manuscript were funded by Merck \& Co. Inc. MH, JP, TB, FXL and RX are employees of Merck \& Co. Inc., the sponsor of this study and manuscript. YL provides advisory and consultancy services to Merck and BMS. VV provides advisory and consultancy services to Merck, BMS, Genentech, AstraZeneca and Celgene. VV has received grants and honoraria from Merck, BMS, Genentech, Heat Biologics, Altor Biosciences, Foundation Medicine and Clovis. 
Open Access This article is distributed under the terms of the Creative Commons Attribution-NonCommercial 4.0 International License (http://creativecommons.org/licenses/by-nc/4.0/), which permits any noncommercial use, distribution, and reproduction in any medium, provided you give appropriate credit to the original author(s) and the source, provide a link to the Creative Commons license, and indicate if changes were made.

\section{References}

1. GLOBOCAN. Estimated Cancer Incidence, Mortality and Prevelance Worldwide in 2012-Lung Cancer. 2012. http://globocan. iarc.fr/Pages/fact_sheets_cancer.aspx. Accessed 8 Sept 2016.

2. Jemal A, Bray F, Center MM, Ferlay J, Ward E, Forman D. Global cancer statistics. CA Cancer J Clin. 2011;61(2):69-90.

3. Cancer Research UK. Lung cancer incidence statistics. 2016. http://www.cancerresearchuk.org/health-professional/cancer-stati stics/statistics-by-cancer-type/lung-cancer/incidence\#headingThree. Accessed 8 Sept 2016.

4. World Health Organization (WHO): Non-small cell lung cancer. 2014. http://www.who.int/selection_medicines/committees/ex pert/20/applications/NonSmallCellLungCancer.pdf. Accessed 8 Sept 2016.

5. US Department of Health and Human Services. https://www.fda. gov/drugs/informationondrugs/approveddrugs/ucm526430.htm. Accessed 26 May 2017.

6. Partitioned Survival Model [online]. (2016). York; York Health Economics Consortium; 2016. http://www.yhec.co.uk/glossary/ partitioned-survival-model/. Accessed 26 May 2017.

7. Reck M, Rodriguez-Abreu D, Robinson AG, et al. Pembrolizumab versus chemotherapy for PD-L1-positive non-smallcell lung cancer. N Engl J Med. 2016;375(19):1823-33.

8. Eisenhauer EA, Therasse P, Bogaerts $J$, et al. New response evaluation criteria in solid tumours: revised RECIST guideline (version 1.1). Eur J Cancer. 2009;45:228-47.

9. FDA KEYTRUDA Prescribing Information. 2016. https://www. accessdata.fda.gov/drugsatfda_docs/label/2016/125514s012lbl. pdf. Accessed 26 May 2017.

10. Sanders GD, Neumann PJ, Basu A, Brock DW, Feeny D, Krahn M, Kuntz KM, Meltzer DO, Owens DK, Prosser LA, Salomon JA. Recommendations for conduct, methodological practices, and reporting of cost-effectiveness analyses: second panel on cost-effectiveness in health and medicine. JAMA. 2016;316(10):1093-103.

11. Latimer NR. NICE DSU technical support document 14: survival analysis for economic evaluations longside clinical trials-extrapolation with patient-level data. London: NICE; 2011.

12. Latimer NR, Abrams KR. NICE DSU Technical Support Document 16: Adjusting survival time estimates in the presence of treatment switching. London: NICE. http://www.nice.dsu.org.uk/ TSD16_Treatment_Switching.pdf.

13. Latimer NR. Survival analysis for economic evaluations alongside clinical trials-extrapolation with patient-level data: inconsistencies, limitations, and a practical guide. Med Decis Mak. 2013;33(6):743-54.

14. Chow GC. Chow tests. 2011. http://www.stata.com/support/faqs/ statistics/chow-tests/. Accessed 13 Oct 2016.

15. Chow GC. Tests of equality between sets of coefficients in two linear regressions. Econometrica. 1960;28(3):15.

16. National Cancer Institute. National Cancer Institute SEER Data, 1973-2013. http://seer.cancer.gov/data. Accessed 13 Oct 2016.

17. Flatiron Health. http://www.flatiron.com/life-sciences. Accessed 8 Sept 2016.
18. Shaw JW, Johnson JA, Coons SJ. US valuation of the EQ-5D health states: development and testing of the D1 valuation model. Med Care. 2005;43:203-20.

19. Dolan P. Modeling valuations for EuroQol health states. MedCare. 1997;35:1095-108.

20. Greiner W, Weijnen T, Nieuwenhuizen $M$, et al. A single European currency for EQ-5D health states. Results from a sixcountry study. Euro. J Health Econ. 2003;4:222-31.

21. Hatswell AJ, Pennington B, Pericleous L, Rowen D, Lebmeier M, Lee D. Patient-reported utilities in advanced or metastatic melanoma, including analysis of utilities by time to death. Health Qual Life Outcomes. 2014;12:140.

22. Huang M, Pellissier J, Kong F. A Trial-Based Euroqol Eq-5d Health Utility Analysis in Patients with Previously Untreated Metastatic NSCLC. In: ISPOR 22nd annual international meeting; Boston, MA; May 20-24, 2017.

23. Center for Medicare and Medicaid Services. Medicare 2016 costs at a glance. https://www.cms.gov/apps/ama/license.asp?file=/ Medicare/Medicare-Fee-for-Service-Payment/HospitalOutpatient PPS/Downloads/CMS-1633-FC-2016-OPPS-FC-Cost-Statistics. zip. Accessed 16 Jan 2017.

24. Bureau of Labor Statistics Consumer Price Index-All Urban Consumers. 2016. https://www.bls.gov/cpi/ Accessed 16 Jan 2017.

25. Analysource. www.Analysource.com. Accessed 8 Sept 2016.

26. NCCN Clinical Practice Guidelines in Oncology: Version 1.2017. http://www.vitromolecularlaboratories.com/wp-content/uploads/ 2016/10/NCCN-1.2017-NSCLC-Guidelines.pdf. Accessed 26 May 2017.

27. Huang M, Pellissier J, Liao J. Cost-effectiveness of pembrolizumab vs. standard-of-care chemotherapy in 1st line treatment of PD-L1 positive metastatic NSCLC patients. https://www. nccn.org/professionals/meetings/22ndannual/pdf/AC2017_Exhi bitGuide.pdf. Accessed 1 May 2017.

28. Center for Medicare and Medicaid Services, Cost Procedural Terminology, Fourth Edition. Costs for Hospital Outpatient Services, by HCPCS code for CY 2016. 2016.

29. Ramsey S, Henk HJ, Smith GL, Sollano J, Chen C. First-, secondand third-line lung cancer treatment patterns and associated costs in a US healthcare claims database. Lung Cancer Manag. 2015;4(3):131-43.

30. Vera-Llonch M, Weycker D, Glass A, Gao S, Borker R, Barber B, Oster G. Healthcare costs in patients with metastatic lung cancer receiving chemotherapy. BMC Health Serv Res. 2011;11:305.

31. Davis KL, Goyal RK, Able SL, Brown J, Li L, Kaye JA. Realworld treatment patterns and costs in a US Medicare population with metastatic squamous non-small cell lung cancer. Lung Cancer. 2015;87(2):176-85.

32. Yabroff KR, Lamont EB, Mariotto A, Warren JL, Topor M, Meekins A, Brown ML. Cost of care for elderly cancer patients in the United States. J Natl Cancer Inst. 2008;100(9):630-41.

33. Lang K, Marciniak MD, Faries D, Stokes M, Buesching D, Earle C, Treat J, Babineaux S, Morissette N, Thompson D. Costs of first-line doublet chemotherapy and lifetime medical care in advanced non-small-cell lung cancer in the United States. Value Health. 2009;12(4):481-8.

34. Chastek B, Harley C, Kallich J, Newcomer L, Paoli CJ, Teitelbaum AH. Health care costs for patients with cancer at the end of life. J Oncol Pract. 2012;8(6):75s-80s.

35. Fox KM, Brooks JM, Kim J. Metastatic non-small cell lung cancer: costs associated with disease progression. Am J Manag Care. 2008;14(9):565-71.

36. Au DH, Udris EM, Fihn SD, McDonell MB, Curtis JR. Differences in health care utilization at the end of life among patients 
with chronic obstructive pulmonary disease and patients with lung cancer. Arch Intern Med. 2006;166(3):326-31.

37. Kutikova L, Bowman L, Chang S, Long SR, Obasaju C, Crown WH. The economic burden of lung cancer and the associated costs of treatment failure in the United States. Lung Cancer. 2005;50(2):143-54.

38. Center for Medicare and Medicaid Services. 2016. https://www.cms. gov/Medicare/Medicare-Fee-for-Service-Payment/AcuteInpatient PPS/FY2016-IPPS-Final-Rule-Home-Page.html Accessed 17 July 2016.

39. Vemer P, Corro Ramos I, van Voorn GA, Al MJ, Feenstra TL. AdViSHE: a validation-assessment tool of health-economic models for decision makers and model users. Pharmacoeconomics. 2016;34(4):349-61.

40. Huang M, Lou Y, Pellissier J, Burke T, Liu FX, Xu R, Velcheti V. Cost-effectiveness of pembrolizumab versus docetaxel for the treatment of previously treated PD-L1 positive advanced NSCLC patients in the United States. J Med Econ. 2017;20(2):140-50.

41. Braithwaite RS, Meltzer DO, King JT, Leslie D, Roberts MS. What does the value of modern medicine say about the $\$ 50,000$ per quality-adjusted life-year decision rule? Med Care. 2008;46(4):349-56.

42. World Health Organization (WHO). Choosing interventions that are cost-effective. http://www.who.int/choice/en/. Accessed 13 July 2016.

43. KNOEMA. IMF World Economic Outlook (WEO). https:// knoema.com/pjeqzh/gdp-per-capita-by-country-1980-2014. Accessed 13 July 2016.

44. Neumann PJ, Cohen JT, Weinstein MC. Updating cost-effectiveness-the curious resilience of the 50,000-per-QALY threshold. N Engl J Med. 2014;371(9):796-7.

45. Institute for Clinical and Economic Review. Overview of the ICER value framework and proposals for an update for 2017-2018. http://
icer-review.org/wp-content/uploads/2016/02/ICER-VAF-UpdateProposals-020117.pdf. Accessed 18 Jan 2017.

46. Beca J, Hoch JS. Exploring the impact of structural uncertainty in partitioned survival models for oncology. Value Health. 2014;17(3):A205-6.

47. Coyle D, Coyle K. The inherent bias from using partitioned survival models in economic evaluation. Value Health. 2014;17(3):A194.

48. Goeree R, Villeneuve J, Goeree J, Penrod JR, Orsini L, Tahami Monfared AA. Economic evaluation of nivolumab for the treatment of second-line advanced squamous NSCLC in Canada: a comparison of modeling approaches to estimate and extrapolate survival outcomes. J Med Econ. 2016;19(6):630-44.

49. National Institute for Health and Clinical Excellence (NICE). NICE Single Technology Appraisal: Evidence review Group Report. Nintedanib for previously treated locally advanced or metastatic non-small cell lung cancer. London: NICE; 2014. https://www.nice.org.uk/guidance/ta347.

50. National Institute for Health and Clinical Excellence (NICE). NICE Single Technology Appraisal. Nivolumab for previously treated locally advanced or metastatic squamous non-small-cell lung cancer. Committee Papers. London: NICE; 2015. https:// www.nice.org.uk/guidance/indevelopment/gid-tag506/ documents.

51. Abernethy AP, Arunachalam A, Burke T, Mckay C, Cao X, Sorg $\mathrm{R}$, Carbone D. Real-world first-line treatment and overall survival in non-small cell lung cancer without known EGFR mutations or ALK rearrangements in US community oncology setting. PLoS One (accepted).

52. Wu E, Liu F, Lou Y, Huang M, Arunachalam A. Budget impact analysis of Keytruda for non-small cell lung cancer: A U.S. payer perspective. J Manag Care Specialty Pharm. 2017;23(3-a):S79. 